\title{
A Case Report of a Repair of a Ruptured Incisional Hernia Using Polypropylene Mesh and Component Separation Technique \\ A Rambhajan, T Bernard
}

\begin{abstract}
Incisional hernias are a common complication of laparotomies and present a challenging surgical problem. The potentially fatal complication of spontaneous rupture and evisceration is rare and requires urgent surgical repair. Intraoperative decision making requires careful assessment of all patient factors and experience with advanced hernia repair techniques. Oftentimes, these hernias are giant sized and there is associated atrophy and ulceration of the overlying skin with a stretched and weakened anterior abdominal wall. Spontaneous rupture and evisceration has been most commonly described in patients with end-stage liver disease and ascites which makes surgery hazardous in this subset of patients. Surgical technique must minimize the occurrence of complications as well as hernia recurrences in these challenging hernias and the surgeon must decide on the use of mesh, the type of mesh, its position and also the use of additional techniques such as component separation. We present a case of the spontaneous rupture of a giant incisional hernia with evisceration of small intestine that was successfully repaired using polypropylene mesh and component separation.
\end{abstract}

Keywords: Component separation, evisceration, incisional hernia, repair, rupture

From: Department of Surgery, Port-of-Spain General Hospital, Trinidad.

Correspondence: Dr Bernard, Department of Surgery, Port-of-Spain General Hospital, Trinidad. \#13 Seventh Street East, Cazabon Avenue, Trincity. E-mail: bernard.tricia@gmail.com Trinidad. 


\section{INTRODUCTION}

Incisional hernias are very common $(1,2)$, however the potentially fatal complication of spontaneous rupture and evisceration is relatively rare (3-7). Management of this complication requires urgent surgical intervention to prevent bowel ischaemia and perforation. Most reports are in patients with liver failure, ascites and umbilical hernias $(5,8,9)$ in whom operative repair may precipitate decompensated liver failure. Definitive repair of the hernia with the use of mesh will result in the best outcome $(1,10)$, once the patient's overall condition allows this.

\section{CASE REPORT}

This is the case of an 80-year-old obese (BMI 30), hypertensive female who had undergone an upper midline laparotomy for bilateral staghorn calculi 14 years prior, with the development of an incisional hernia within one year of that operation. She had been advised, by her primary institution, on non-surgical management of her large hernia.

The patient presented to hospital acutely with a $20 \mathrm{~cm}$ loop of small intestine having eviscerated through a $2 \mathrm{~cm}$ defect in the skin of the most inferior aspect of the hernia at the umbilicus (Fig. 1). 


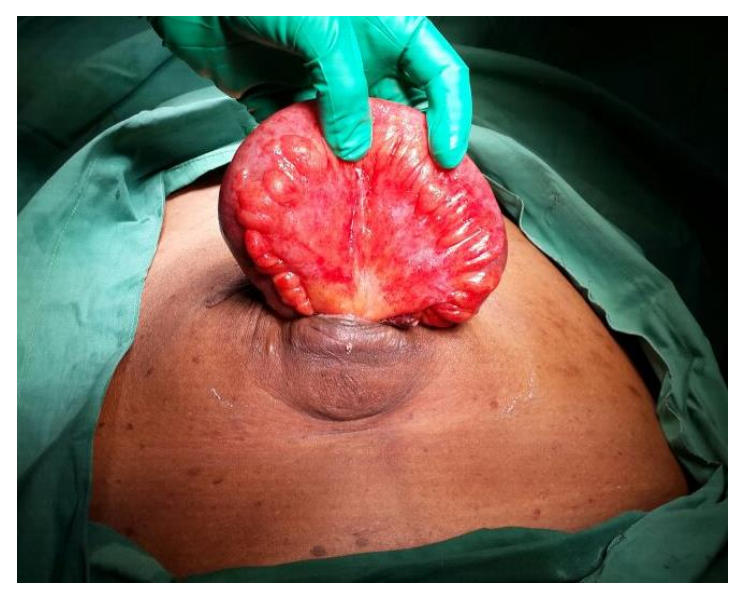

Fig: $1.20 \mathrm{~cm}$ loop of small intestine eviscerated through $2 \mathrm{~cm}$ defect in skin at umbilicus.

She reported constipation during the week prior to admission, with rupture occurring while rising from a supine position. The bowel was kept moist and the patient was taken urgently to the operating theatre for repair under general anaesthesia.

The hernia sac and skin were fused in the area of the evisceration. The small intestine appeared healthy, with no need for resection. All omental adhesions were dissected free off the fascial edges and a large Swiss cheese defect in the fascia was unveiled. The defect (Fig. 2) measured 20 x $25 \mathrm{~cm}$.

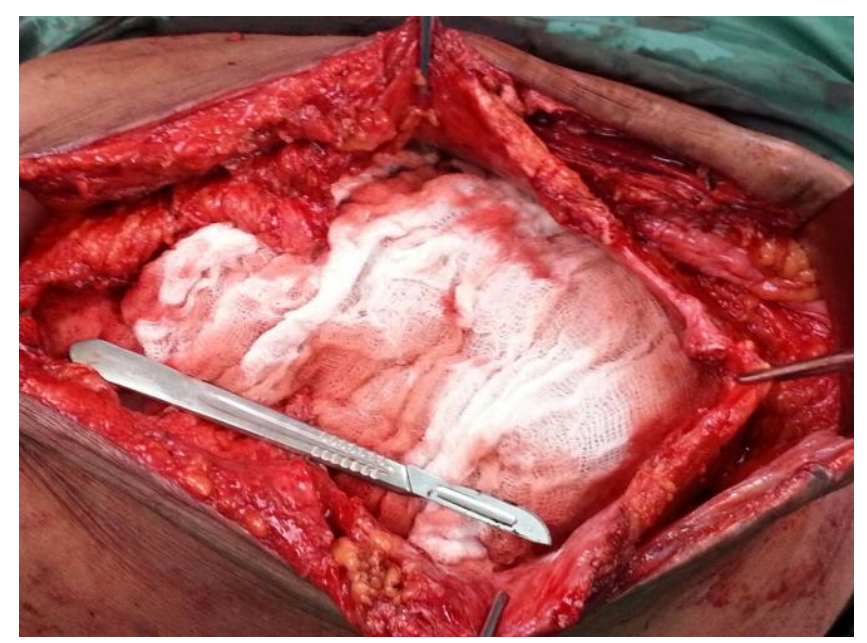

Fig: $2.20 \mathrm{~cm} \times 25 \mathrm{~cm}$ fascial defect seen after dissection of hernia sac. 
The subcutaneous tissue was circumferentially dissected off the rectus sheath. An anterior component separation was performed by making a longitudinal incision along the external oblique aponeurosis, $2 \mathrm{~cm}$ lateral to the rectus abdominis (Fig. 3). The external oblique aponeurosis was separated from the underlying internal oblique muscle. This medialised the rectus sheath and allowed a tension free hernia repair.

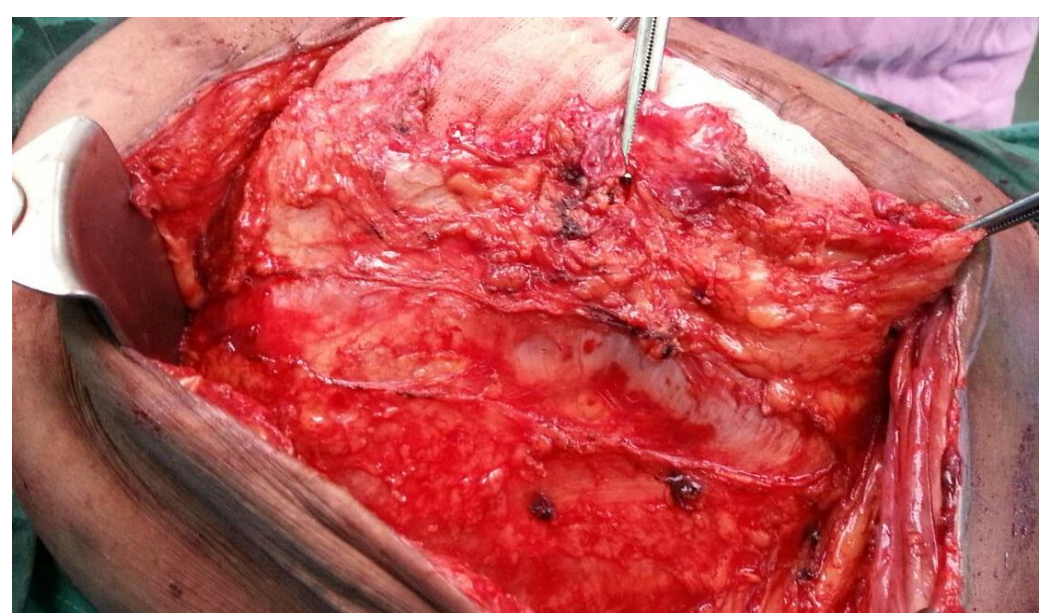

Fig: 3. Anterior component separation.

Primary repair was performed using interrupted 0 -Nylon sutures and an onlay polypropylene mesh (Fig. 4).

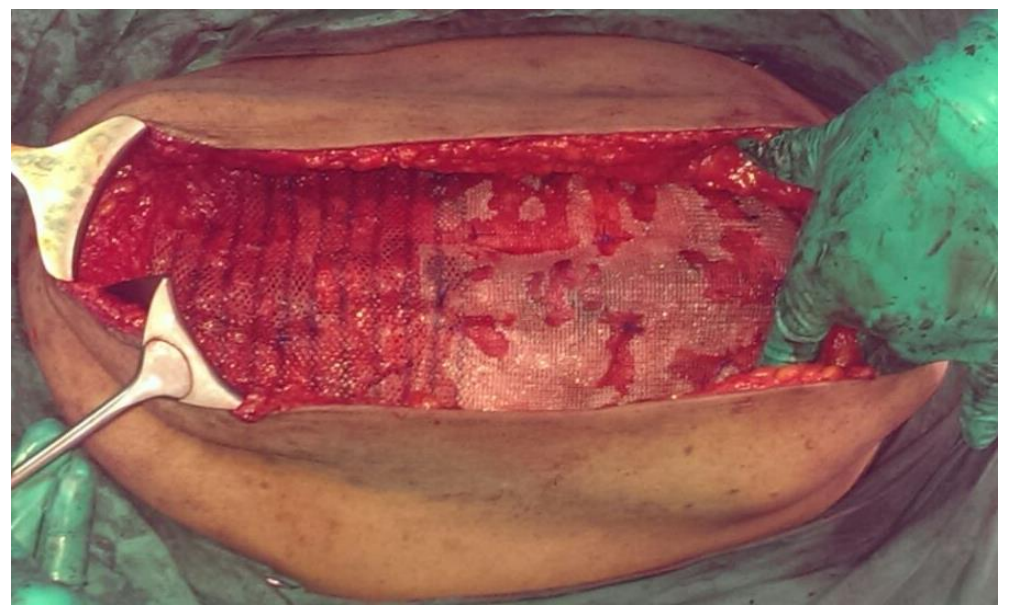

Fig: 4. Onlay polypropylene mesh. 
Any redundant atrophic skin was sharply excised and two 15Fr Blake drains were placed in the suprafascial space prior to skin closure. The operative time was 135 minutes and the estimated blood loss was $100 \mathrm{~mL}$. The patient recovered without event and was discharged to home on the third postoperative day and went on to have satisfactory wound healing and a good cosmetic outcome.

\section{DISCUSSION}

Incisional hernias are very common, and occur in up to $22 \%$ of patients after a midline laparotomy (1). This poses a significant surgical burden and some hernias may require advanced techniques to achieve repair. Recurrence continues to be of high concern with rates of up to $63 \%$ without the use of mesh $(2,10,11)$. With frequent wound complications, chronic abdominal pain and poor cosmesis, patient dissatisfaction is also an issue (11). These factors may act as deterrents for surgeons to repair asymptomatic hernias.

Spontaneous rupture and evisceration is often precipitated by a bout of constipation and straining at stool $(4,7)$ or coughing $(3,6,8,9)$. Rupture has also been reported with simply turning in bed (5). It is also associated with chronic dermatitis and excoriation of the skin of the hernia sac with formation of an ulcer prior to rupture $(3-5,7)$. Other reports of spontaneous rupture of hernias with evisceration have been associated with massive ascites and liver failure $(8,9)$. Our patient's acute presentation was preceded by a bout of constipation along with skin atrophy which had gone unnoticed.

Our patient's surgery was a technical challenge owing to her giant [>15cm] (11) hernia defect, the longstanding nature of the hernia which caused her rectus abdominis muscles to 
retract laterally and her acute presentation. A simple approximation of the fascia in the midline would have produced a suture line under tension. For this reason, a component separation was performed to assist in the medialisation of the rectus muscles and relieve the tension from the suture line, using autologous tissue. Fascial component separation is a useful adjunct to incisional hernia repair, and is especially useful in recurrent hernias, or those that occur in the obese population $(11,12)$. Moore et al reported a recurrence rate of $5.5 \%$ when using a similar component separation technique on obese patients (12).

An onlay placement of polypropylene mesh with large overlap was performed to reduce the chance of a hernia recurrence $(2,11)$. A 2011 Cochrane review (10) found insufficient evidence to determine whether the onlay or sublay position of mesh in incisional hernia repair was superior. Subsequent authors have recommend the sublay position because of a "trend towards lower recurrence rates" (13) and a lower incidence of wound complications $(11,14)$. With our patient, no other method of mesh placement was possible because composite mesh was unavailable, and dissection of the preperitoneal space was not possible as the hernia sac was firmly adherent to the overlying skin along the midline incision. The safe use of mesh in this type of acute presentation has previously been described in two case reports $(3,8)$.

Spontaneous rupture of a hernia with evisceration is a rare occurrence and can be challenging to manage. We present the first reported case in the literature where this was addressed by immediate definitive repair with an anterior component separation and an onlay of polypropylene mesh.

\section{ACKNOWLEDGEMENTS}

The authors declare that there are no other persons to acknowledge for the composition of this case report. 


\section{REFERENCES}

1. Fink C, Baumann P, Wente M, Knebel P, Bruckner T, Ulrich A et al. Incisional hernia rate 3 years after midline laparotomy. Br J Surg 2014; 101: 51-4.

2. Burger J, Luijendijk R, Hop W, Halm J, Verdaasdonk E, Jeekel J. Long-term follow-up of a randomized controlled trial of suture versus mesh repair of incisional hernia. Ann Surg 2004; 240: 578-85.

3. Martis J, Shridhar K, Rajeshwara K, Janardhanan D, Jairaj D. Spontaneous rupture of incisional hernia — a case report. Indian J Surg 2011; 73: 68-70.

4. Friedman MHW. Spontaneous evisceration through a ventral hernia. Canad Med Ass J 1965; 92.

5. Ginsberg BY, Sharma AN. Spontaneous rupture of an umbilical hernia with evisceration. J Emerg Med 2006; 30: 155-57.

6. Gupta RK, Sah S, Agrawal SC. Spontaneous rupture of incisional hernia: a rare cause of a life-threatening complication. BMJ Case Rep 2011; doi:10.1136/bcr.11.2010.34862

7. Zaz MA, Dass T, Muhee A, Kawoosa U. An unusual case of inguinal hernia with spontaneous evisceration. Hernia 2011; 15: 705-07.

8. Ogu US, Valko J, Wilhelm J, Dy V. Spontaneous evisceration of bowel through an umbilical hernia in a patient with refractory ascites. J Surg Case Rep 2013;10 doi:10.1093/jscr/rjt073

9. Choo EK, McElroy S. Spontaneous bowel evisceration in a patient with alcoholic cirrhosis and an umbilical hernia. J Emerg Med 2008; 34: 41-43. 
10. Den Hartog D, Dur AH, Tuinebreijer WE, Kreis RW. Open surgical procedures for Incisional hernias. Cochrane Database Syst Rev 2011; CD006438.

11. Eriksson A, Rosenberg J, Bisgaard T. Surgical treatment for giant incisional hernia: a qualitative systematic review. Hernia 2014; 18: 31-38.

12. Moore M, Bax T, MacFarlane M, McNevin MS. Outcomes of the fascial component separation technique with synthetic mesh reinforcement for repair of complex ventral incisional hernias in the morbidly obese. Am J Surg 2008; 195: 575-9.

13. Timmermans L, De Goede B, Van Dijk S, Kleinrensink G, Jeekel J, Lange J. Metaanalysis of sublay versus onlay mesh repair in incisional hernia surgery. Am J Surg 2014; 207: $980-88$.

14. Venclauskas L, Maleckas A, Kiudelis M. One-year follow-up after incisional hernia treatment: results of a prospective randomized study. Hernia 2010; 14: 575-82. 